\title{
Evoked Potentials
}

\author{
Ahmet Akay \\ Ege University \\ Turkey
}

\section{Introduction}

Evoked potentials are electrical activities that occur in the neural pathways and structures as a response to various external stimulations induced by light, sound, electric, smell, or taste. Evoked potentials are polyphasic waves that oftenly present with an amplitude between 0.1$20 \mu \mathrm{A}$ which are formed within $2-500 \mathrm{~ms}$. The source of these activities is probably the summation of the action potentials generated by the afferent tracts and the electrical fields or activities of the synaptic discharges or post-synaptic potentials on those tracts.

Understanding evoked potentials bears importance in terms of controlling the entire pathway from stimulation point to the cortical areas, in other words, to the primary cortex. By examining evoked potentials, we can find answers to many questions such as: Does the response against the stimulus reach intended destinations on time? Does the response show any loss of intensity? If there is a problem in the neural parthways, what is its exact location?

When a person receives a visual stimulus such as a flash light, the EEG (electroencephalogram) record concerning the occipital region (particularly at the O1-P3 and $\mathrm{O} 2-\mathrm{P} 4$ derivations) demonstrates waves that are called "photic driving response" which form within about $150 \mathrm{~ms}$ and can be observed after any kind of light stimulation. However, most of the responses elicited due to external stimulation are not observed very clearly because those tiny little responses that we call "evoked potentials" can not be seen in EEG signals which may have amplitudes reaching $100 \mu \mathrm{V}$.

Moreover, there are non-neural activities that suppress those signals such as EEG: ECG (electrocardiogram), EMG (electromyogram), and other biologic signals. In addition, when the noises of the electronic devices and the environment are also considered, the difficulty of isolating evoked potentials can be figured out more easily. Currently, there are two methods used for eliminating those external interferences and both of them should be used in combination: "filtration" and "averaging".

Today, novelties in the electronics and computerized devices present us these advantages: High-quality amplifiers, smaller devices, perfect averaging techniques, multichannel capability, quality filtration options (digital filter, adaptive filter, spectral analysis etc.), capability of recording in any kind of environment (eg. operating room, bedside, or noisy environments), routine use of evoked potential recording systems in clinics. 
The three major types of evoked potentials used in clinical studies are visual evoked potentials (VEP), brainstem auditory evoked potentials (BAEP), and somatosensory evoked potentials (SSEP).

\section{Recording sytem}

\subsection{Electrodes}

In evoked potential recordings, $\mathrm{Ag} / \mathrm{AgCl}$ or gold-plated surface electrodes with a hole are used. Needle electrodes should not be employed because they produce many artifacts and have high electrode-skin impedance (5000-7000 $\Omega$ ). The electrode-skin impedance in surface electrodes is lower than $5000 \Omega$, within a range of 2000-3000 $\Omega$ (under intensive care settings or in comatose patients and during intraoperatif use, needle electrodes or disposable electrodes can be employed for following the evoked potentials).

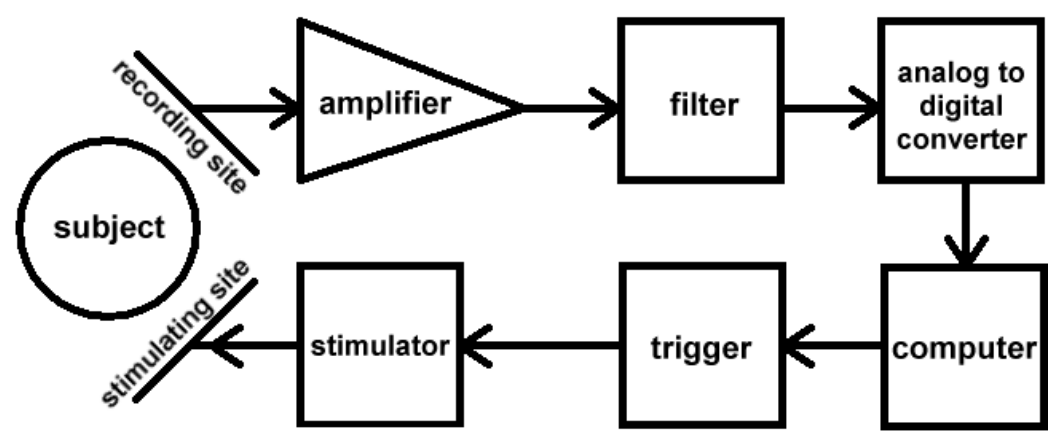

Fig. 1. Block diagram of the recording system.

Nonetheless, surface electrodes and needle electrodes do not have any difference with regard to recording quality of evoked potentials, both in terms of amplitude and waveform. In addition to the use of chloride gel in all electrodes, collodion is applied on hairy skin areas (for fixation purposes in long recordings).

Prior to the placement of the electrodes, the related skin surface should be cleaned with alcohol or acetone in order to adequately reduce the electrode-skin impedance. In case of need, abrasive gels can be applied for this purpose.

During recording, bipolar electrodes are preferred because lowest noise level can be achieved only by them. As the distance between the bipolar electrodes increase, signals grow more strong, however, the noise increases by the same rate, as well.

Stimulus electrodes are also bipolar and the distance between them should be $35 \mathrm{~mm}$ with the active electrode placed distally.

The electrode configuration and system of a classic 10/20 electrode EEG recording system, shown in the Figure 2, are used in evoked potential recordings, as well. However, if needed, the electrode configuration of a 32-electrode EEG recording system can also be employed. (F: Frontal region, C: Central region, T: Temporal region, P: Parietal region, O: Occipital region, A1 and A2: Ear lobes, Fz, Cz, Pz, Oz: Midline electrodes, The odd numbered electrodes are always placed on the left.) 

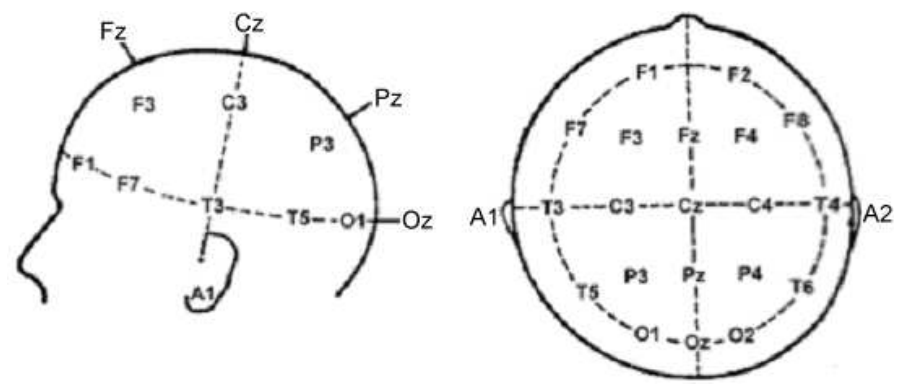

Fig. 2. International 10/20 EEG electrode positioning system.

\subsection{Amplifier}

In evoked potential recordings, differential amplifiers that can carry out 500,000x amplification which can be intensified stepwise, are used (an amplifier that amplifies the difference between voltages of the first and second inputs). One of the main characteristics of differential amplifiers is the capability of eliminating electrostatic and electromagnetic interferences from reaching the patient.

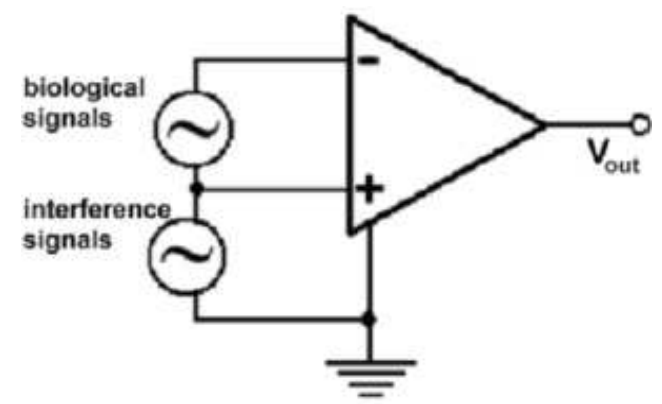

Fig. 3. Two main signals in the input of an amplifier.

The input of the amplifier has components of 6 different signals:

1. The biological signal that we aim to measure (evoked potential)

2. The biological signals that we do not want to measure (cortical, cardiac, muscular, and other biological activities)

3. $50 \mathrm{~Hz}$ interference signal and its harmonics

4. Interference signals arising from the electrode-tissue contact

5. Environmental interferences (electromagnetic, electrostatic, radio-frequency interferences)

6. Noise of the amplifier itself.

\subsection{Filter circuit}

It comprises serial connection of a low-cut filter (high-pass filter) and a high-cut filter (lowpass filter) including passive and active components, or a band-pass filter bearing the characteristics of those two filters. 


\begin{tabular}{|c|c|c|}
\cline { 2 - 3 } \multicolumn{1}{c|}{} & Low-cut & High-cut \\
\hline SSEP & $30 \mathrm{~Hz}$ & $3000 \mathrm{~Hz}$ \\
\hline BAEP & $100 \mathrm{~Hz}$ & $3000 \mathrm{~Hz}$ \\
\hline VEP & $0.2-1 \mathrm{~Hz}$ & $200-300 \mathrm{~Hz}$ \\
\hline
\end{tabular}

Table 1. Filter values used in evoked potential recording systems.

Notch filters which is applied for eliminating $50 / 60 \mathrm{~Hz}$ mains electricity interference, is not used in any of the evoked potential recording procedures in order to not eliminate the 50/60 $\mathrm{Hz}$ components of the concerning evoked potential signals (Since low-cut frequency for BAEP is already $100 \mathrm{~Hz}$, there is no need for $50 / 60 \mathrm{~Hz}$ notch filter) Instead of high-cut and low-cut filters, band-pass filters of 30-3000 Hz for SSEP, 100-3000 Hz for BAEP, and (0.2-1)(200-300) Hz for VEP, can be used.

As shown in the Figure 1, filter circuit can be placed at the amplifier output or between the preamplifier stage and power amplifier stage. However, the best is to use a low-cut filter at the pre-amplifier output and a high-cut filter at the power amplifier output.

\subsection{Analog-to-digital converter}

Because computers can only work with numbers, the voltage changes at the filter output should be converted to digital data. Thus, analog-to-digital converter (ADC) performs this function. An analog-to-digital converter comprises "sampler" and "quantizer" circuits (Figure 4).

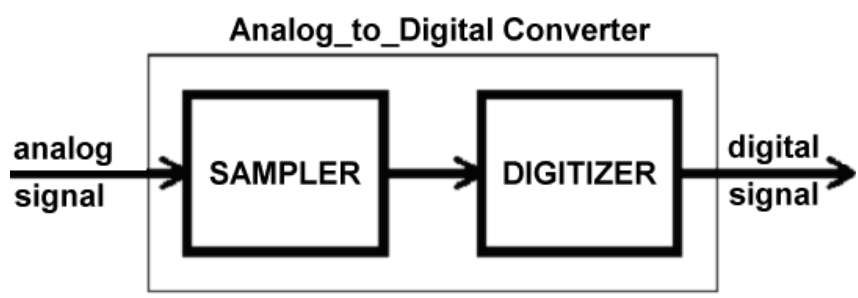

Fig. 4. Inside of the ADC circuit.

These two circuits should enable numerical ADC output, in other words "time series", to represent the original signal (the evoked potential we are trying to generate) in a complete way and thus carry the data included within the signal without a loss. In short, "sampling" and "quantization" ranges should be chosen sufficiently small (The vertical resolution of the ADC circuit, ie. "voltage resolution", should be lower than the least amplitude value of the signal; whereas its horizontal resolution, ie. temporal resolution, should be at least twice the highest frequency component) (Figure 5).

For example, lets say, the sampling sensitivity of our ADC circuit is 12-bit and the reference voltage is $\pm 5 \mathrm{~V}$. In this case, the sampling range will be $(5+5)$ volt $/ 2^{12}=2 \mathrm{mV}$, which indicates that the circuit will detect the amplitude changes $\geq 2 \mathrm{mV}$. Since an amplitude value of $0.1 \mu \mathrm{V}$ in an evoked potantial recording can reach a value of $10 \mathrm{mV}$ after undergoing 100,000x amplification, an ADC circuit of 12-bit can easily be detected and converted to a digital value. However, if we use an 8-bit ADC circuit, because the sampling range of this 


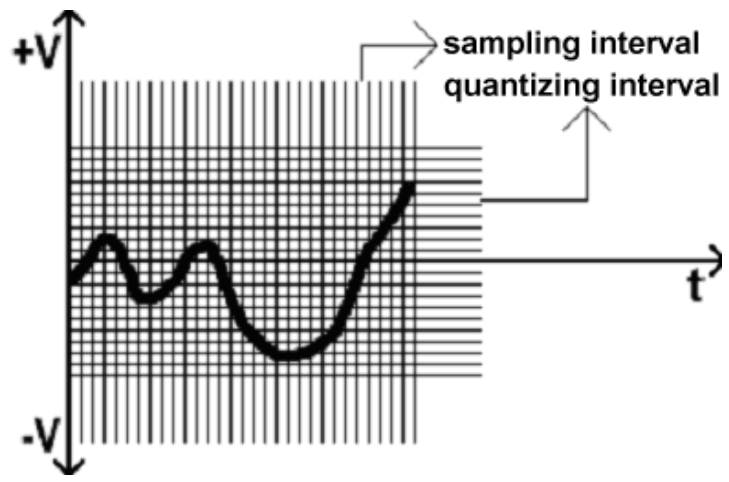

Fig. 5. Sampling \& quantizing intervals of a signal.

would be $(5+5)$ volt $/ 2^{8}=39 \mathrm{mV}$, a $0.1 \mu \mathrm{V}$ change in the evoked potential wave or amplitude will not be detected, and thus will be eliminated.

As you can see in our example, the sampling sensitivity of ADC circuits used in recording evoked potentials should be 12-bit. Therefore, 12-bit ADC circuits are the standard in this regard.

Lets give another example. If we have an ADC circuit with a sampling sensitivity of 1000 $\mathrm{Hz}$, the subsequent quantization range will be $1 / 1000=1 \mathrm{~ms}$, meaning that as a result of the Nyquest formula, our ADC circuit will not detect evoked potential changes faster than $2 \times(1$ $\mathrm{ms})=2 \mathrm{~ms}$. Because a change of $2 \mathrm{~ms}$ corresponds to $1 / 0.002=500 \mathrm{~Hz}$, in our target evoked potential, the changes faster than $500 \mathrm{~Hz}$ will not be converted into digital data and thus will subsequently vanish.

In short, if the high-cut frequency of our filter circuit is $600 \mathrm{~Hz}$ and the sampling frequency of our ADC circuit is $1000 \mathrm{~Hz}$, it means that we will not be able to see the components of the evoked potential signal between $500-600 \mathrm{~Hz}$. In conclusion, the quantization range used in the ADC circuit depends on the high-cut filter frequency in the filter circuit, and it should be at least twice the value of this frequency.

\subsection{Computer}

Triggers stimulation circuits at appropriate intervals; takes trigger-locked samples of same duration from digital signals elicited from the ADC circuit; after a certain amount of sampling, subjects them to "averaging" process; renders the obtained evoked potentials ready for visualization, evaluation, and storage.

\subsubsection{Triggering}

It is a must that the stimulation circuits are triggered by a computer, in other words, stimulation times should be determined by a computer because the samples should be synchronous with those triggerings, which can be termed as being "trigger-locked". Otherwise, we can not determine the starting time of the samples, and thus fail to evaluate the signals. The samples (epoches) have 3 properties: 
1. Sample duration SSEP: $40 \mathrm{~ms}$ for upper extremities, $60-80 \mathrm{~ms}$ for lower extremities, BAEP: $10 \mathrm{~ms}$, VEP: 200-250 ms.

2. Sample amount SSEP: 500 samples, BAEP: 2000 samples and should be repeated twice, VEP: 200 samples and should be repeated thrice.

3. Onset of sampling After the the samples undergo summation and averaging, the obtained evoked potential signal should start at a certain time relative to the stimulus in order to be valid for evaluation (ie. it should be stimulation-locked). The starting time of the samples may be one of the following three:

At the onset of stimulation The computer starts sampling simultaneously with the triggering for stimulus. This is the most commonly preferred method.

Before stimulation The computer starts sampling prior to the stimulus. In this method, the aim is to see the isoelectric line (to predetermine it). It is applied when amplitudes of evoked potentials are wanted to be measured relative to the isoelectric line or when the events before stimulus are aimed to be determined.

After the stimulation The computer starts sampling after the stimulus. This is the less commonly used method. The aim here is to eliminate any interference of stimulus artifact over the evoked potential.

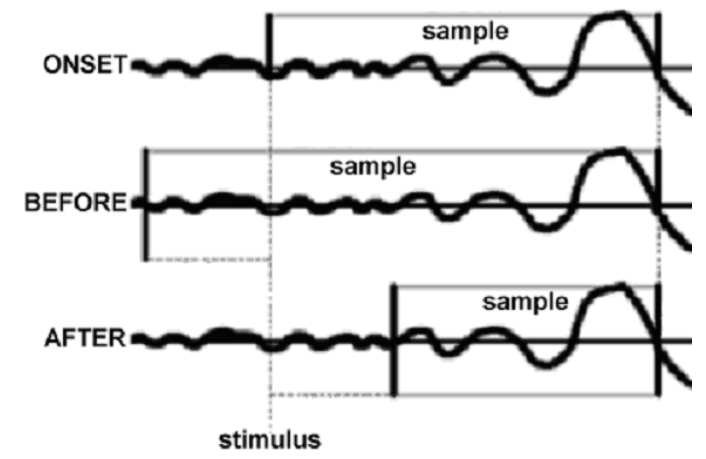

Fig. 6. Three different sampling methods.

\subsubsection{Averaging}

Despite the fact that all EEG signals are generated by the grey matter of the cerebral cortex (reflecting the cortical activity), both cortical and subcortical structures as well as the white matter creating the neural pathways contribute to the evoked potential signals. Since evoked potential signals which can also termed as EEEG (Evoked EEG), have markedly lower amplitude compared to the EEG signals with a normal background activity, they are embedded within the ECG traces and thus they can not be seen directly.

As known by many, EEG signals are spontaneous polyphasic waves whose amplitudefrequency-phase values constantly vary. However, evoked potential signals are changes that are non-spontaneous and have a specific waveform. Moreover, their occurrence times following the stimulus are known. 
Therefore, when we superimpose numerous samples obtained from spontaneous "random" signals such as EEG, the resultant graphic will be an appearance that is entirely filled up to its lower and upper amplitude limits. The averaging of this graphic will produce only "nil", represented by a graphic consisted of an isoelectric line (the upper and lower areas will balance each other)! If we sum up the samples in sequence instead of applying superimposition, we acquire the same result (as shown in the figure below). Thus, first summing up "sample 1" and "sample 2", then "sample 1+2" and "sample 3", then "sample $1+2+3$ " + "sample 4 ", in short, by following the formula of $(n-1)+$ sample $n$, and applying averaging, no signal will be observed in the end ( $\mathrm{n}=$ infinite)!
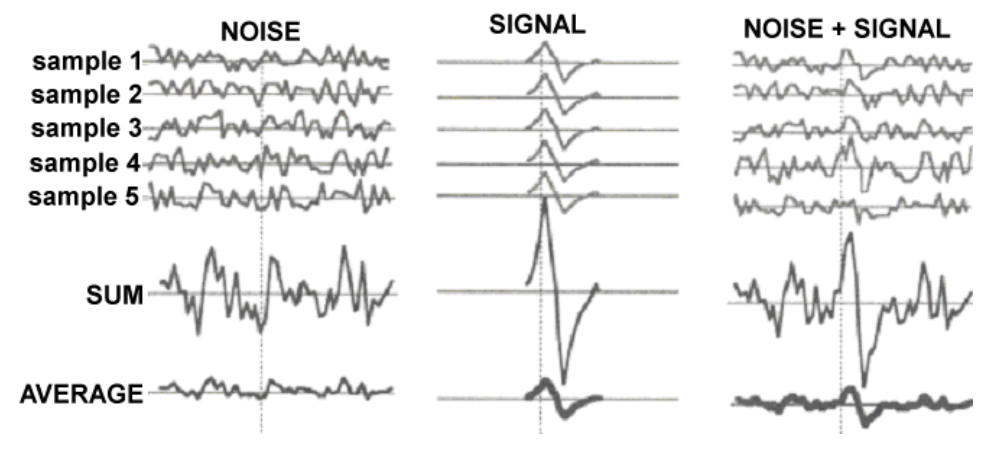

Fig. 7. Averaging of samples.

However, if we perform the same procedure for non-spontaneous and specific evoked potentials, the signal increases at the end of each summation, and finally, the averaging produces the same signal with its original waveform.

When the samples generated by the computers from the evoked potential recordings are subjected to the above mentioned processes, ie. averaging, spontaneous waves such as EEG vanish, whereas the evoked potential wave becomes more significant (as shown in the figure above).

Actually, averaging is a term used for increasing the signal-to-noise ratio ( $\mathrm{S} / \mathrm{N}$ ratio) that is markedly low such as $1 / 50$ or even $1 / 100$. At this point, some may ask the following question: Since it is a fact that infinite number of samples can not be acquired, how many samples should be used in this averaging process aiming to reveal the evoked potential signal?

We can calculate this practically as follows: for example, lets take the average amplitude for EEG waves with background noise as $50 \mu \mathrm{V}$ and take the average amplitude of the related evoked potential as $5 \mu \mathrm{V}$ (as in VEP). The "signal/noise ratio" of this system is $5 \mu \mathrm{V} / 50 \mu \mathrm{V}$ $=0.1$, and we have to mulply it with at least 10 in order to elevate it to 1 . This number that we multiply the result with is termed as "supression factor" and it is shown by $\sqrt{ } \mathrm{N}$. When $\sqrt{ } \mathrm{N}$ is 10 , then $\mathrm{N}$ equals to 100 , which represents the minimal "sample" amount that should be used for averaging.

Again, lets suppose that average amplitude of the EEG waves is $50 \mu \mathrm{V}$, whereas average amplitude of the evoked potential is $0.25 \mu \mathrm{V}$ (as in BAEP). In this case, signal/noise ratio is $0.25 \mu \mathrm{V} / 50 \mu \mathrm{V}=0.005$. We should multiply this value with 200 in order to elevate it to 1 . 
Therefore, suppression factor $(\sqrt{ } N)$ is 200 and $N=4000$. In other words, the minimum number of samples to be used in the BAEP recording should be 4000 .

There is a shortcut formula to find the required minimal sample amount: "Amplitude of the background noise / Amplitude of the evoked potential = Suppression factor" The square root of the result provides us the minimal amount of samples.

\subsection{Stimulation}

First thing that should be reminded here is that the applied frequency should not conflict with the upper or lower harmonics of the mains electricity regardless of the type of the stimulus and the evoked potential test (this is the reason why stimulus frequency is applied as $4.71 / \mathrm{s}$ instead of $5 / \mathrm{s}$ for SSEP, $11 / \mathrm{s}$ instead of $10 / \mathrm{s}$ for BAEP, and $1.98 / \mathrm{s}$ instead of $2 / \mathrm{s}$ for VEP).

\subsubsection{Somatosensory stimulation}

Square-wave stimulation of $10 \mu \mathrm{s}-2 \mathrm{~ms}$ duration is applied (oftenly, square waves with $100-200 \mu$ s are delivered). Stimulus frequency is at most $100 / \mathrm{s}$ (in practical cases, stimulus frequency is $1-5 / \mathrm{s}$ for upper extremities and $0.5-2 / \mathrm{s}$ for lower extremities).

The current intensity of the applied square wave does not exceed $20 \mathrm{~mA}$ and generally occurs between 10-15 mA. However, in medical disorders that increase the stimulation threshold required for generation of an action potential (ie. neural response), this current may need to be elevated (eg. it may be required to elevate it up to $50 \mathrm{~mA}$ in peripheral neuropathy).

Stimulus electrodes should be placed in a way to have a $35 \mathrm{~mm}$ distance to each other, while the active electrode should be placed distally.

\subsubsection{Auditory stimulation}

Square waves of 100-200 $\mu$ s are applied on a single ear through an ear phone (click sound). Simultaneously, the contralateral ear should receive white noise in a continuous fashion (a hissing sound covering all frequencies). Generally 10 stimuli are applied and it is enough that they are of $65-70 \mathrm{~dB}$ intensity.

\subsubsection{Visual stimulation}

It is performed by a TV screen that is $70-100 \mathrm{~cm}$ distant to the patient and having a constant luminance along with checkerboard pattern where the white and black colors are displaced in an alternating fashion. If possible, the test room is preferred to be dark. The alternation rate of the colors is 1 or 2 colors a second (in faster alternations, evoked potential is delayed).

\section{General principles}

Before the test

1. The patient should wash his/her hair on the evening before the test or a couple of hours in advance. 
2. Substances such as perfume, lotion, or cream should not be applied prior to the test.

3. Any accessories such as an auditory device, glasses, or lense should be brought to the clinic.

4. The patient should dress in a comfortable way and clothes with a turtle neck or bra should not be worn.

5. Drugs or foods containing caffeine should not be taken.

6. The people that will undergo visual evoked potential test, should not use sedatives.

During the test

1. In somatosensory, dermatomal, and auditory evoked potential tests, patient is told that the test could take a while and that he/she would take a nap during the test.

2. In somatosensory evoked potential test, the patient should be informed that a tingling can be felt in the stimulation points and flexion may be observed in the thumbs and toes.

3. The patient should be informed that there is no limitation of drug, food, or activity after the test.

4. Skin surfaces that will receive the electrodes should be rubbed with alcohol and acetone, and smoothened if required to.

5. In presence of muscular noise during the auditory evoked potential test and if the patient can not sleep, then sedation with chloride hydrate or diazepam should be performed.

6. Prior to the visual evoked potential test, the visual acuity and pupil width should be controlled in both eyes of the patient.

After the test

There is no limitation of drug, food, or acitivity.

\section{Somatosensory evoked potentials}

They are used for evaluating the synaptic terminals extending towards cortex, by stimulating the peripheral sensory pathways via delivery of an electric current. In short, it is aimed to acquire a response that can be recorded electrically in the central nervous system against a stimulation applied on vibration, position, or epicritic tactile senses.

As noted above, this method, applied for stimulating the central nervous system externally, is a short-term electrical shock that is applied on peripheral (sensory) nerves. Although Eps can be elicited by more natural stimulation methods such as mechanic, tactile, thermic, and pain, they have no practical value as of yet.

If we place the active electrode over the contralateral parietal region and reference electrode over the back of the hand in order to stimulate the median nerve via wrist, and acquire an evoked potential signal while obtaining samples with long duration, we can acquire a polyphasic complex with many ups and downs which starts 9-10 ms after the stimulus and continue until $500 \mathrm{~ms}$.

In Figure 8, the "late components" are nonspesific waves which can vary even in the same individual, depend on the state of attention-consciousness of the individual, and are easily influenced by the used drugs. They occur not only from the primary cortex, ie. post-central 
region, but also as a result of the cortical association, activities of the components, and effects of ARAS (Ascending Reticular Activating System); they are associated with cortical integration.

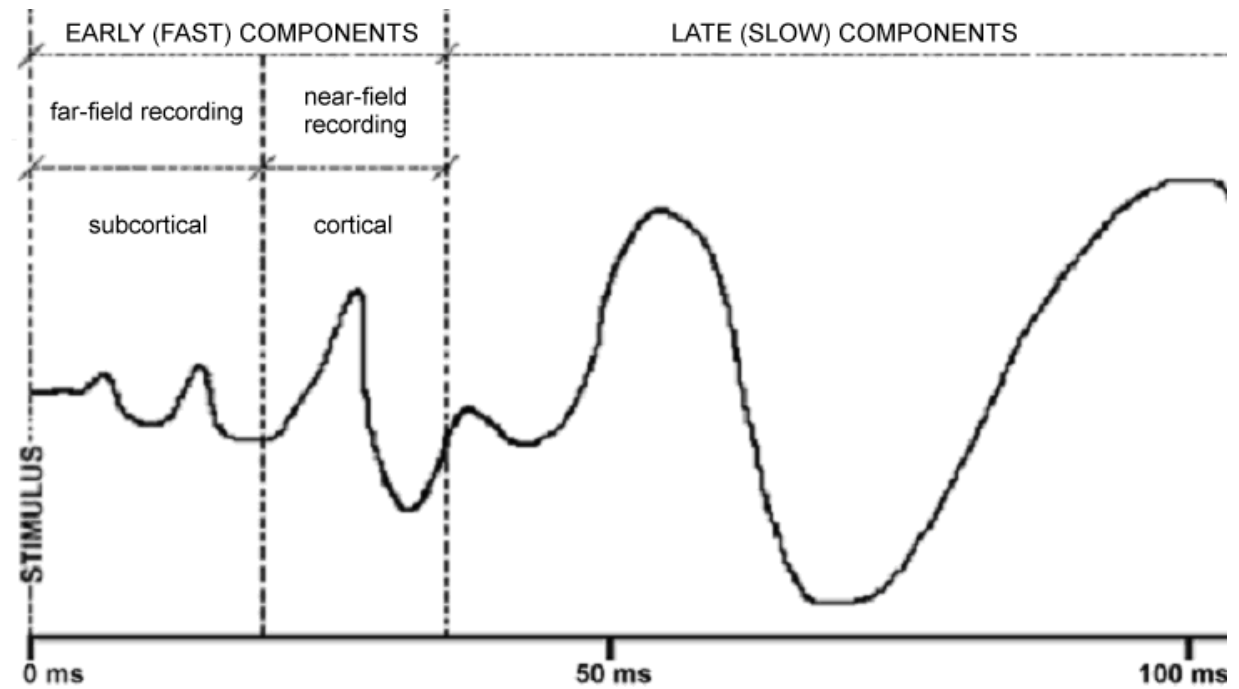

Fig. 8. Components of the SSEP.

Therefore, those late components of SSEP do not have the characteristics of a somatosensory-specific evoked potentials. In studies on evoked potentials, investigators do not focus on these waves, however, they become more important in psychologic, psychiatric, and pharmacologic studies.

When we take a look at the "early components" of the graphic, we see potentials with very small amplitude for 10-15 ms before the occurrence of components with a large amplitude that start nearly $20 \mathrm{~ms}$ after stimulus. Thus, those potentials with a large amplitude are cortical (post-rolandic) activities that are recorded both from under the active electrode and around it, and they are called as "near-field recordings".

On the other hand, small-amplitude waves that occur prior to the near-field potentials, are activities that arise from subcortical structures (brachial, plexus, spinal medulla, lemniscus medialis etc.); they are called as "far-field recordings". In other words, these are evoked potential activities that are located far from the active electrode and as per volume conductivity law, they are detected by and recorded by the active electrode. In short, we can call near-field records as "cortical components" and far-field records as "subcortical components".

Another point that needs explanation here is that there is a classification in the related terminology:

Late components Same as the late components we mentioned above.

Middle components The near-field portion of the above mentioned early components (cortical early component recording). 
Early components The far-field portion of the above mentioned early components (subcortical early component recording).

Since the response generated after the stimulus first passes through the subcortical structures, subcortical components are observed earlier, and because they are located far from the active electrode, they do not have a high amplitude. Their polarity and amplitude may vary depending on the location of the reference electrode, and some components may not appear. For example, when the reference electrode is placed on the forehead, subcortical components become weak and polarities are reversed.

Is there a way to record subcortical components as "near-field" or in other words, can we obtain afferent components in detail? The only way for that is to place the active electrode close to the sources. As shown by Figure 10, if we place three active electrodes separately to the Erb point (ipsilateral), cervical region, and parietal region (contralateral), and carry out a synchronized recording, we can acquire evoked potentials from brachial plexus, spinal medulla, and cortical region separately.

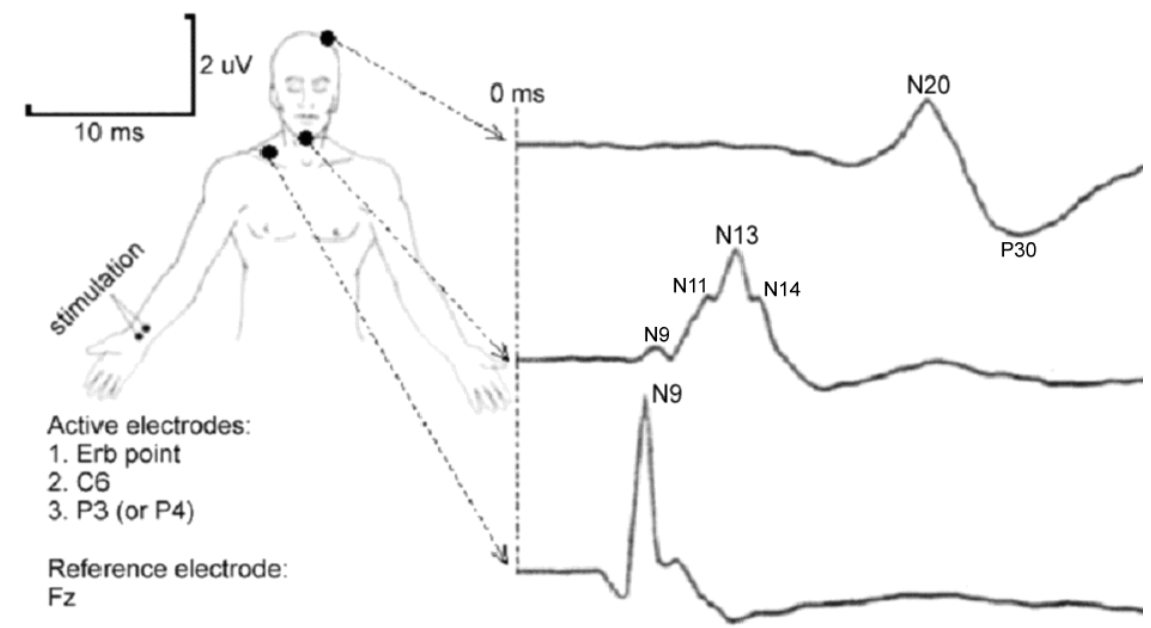

Fig. 9. Peripheral, subcortical, and cortical evoked potentials obtained from three different recording sites.

In clinical practice, active electrodes are P3-P4 or C3-C4 in the upper extremity test and C1$\mathrm{C} 2$ or only $\mathrm{Cz}$ in the lower extremity test; whereas reference electrode is Fz or A1-A2. Again in clinical practice, the most commonly used upper extremity nerves are median and ulnar (at wrist level) nerves, whereas most commonly employed lower extremity nerves are tibial (at ankle level) and peroneal nerves (at knee level).

Spike potential observed in the record obtained from Erb point is named as N9 due to the fact that it is a negative wave occurring nearly $9 \mathrm{~ms}$ after the stimulus. Spike potential observed in the record obtained from cervical region is named as N13 due to the fact that it is a negative wave occurring nearly $13 \mathrm{~ms}$ after the stimulus. The spur on the ascending arm of the N13 is N11 and the spur on the descending arm of the N13 is N14. Moreover, N9 wave can be observed here, as a negative potential just before N11 (far-field record). 
The first wave observed in the record obtained from the parietal region is N20, which is a negative wave that occurs $20 \mathrm{~ms}$ after the stimulus. Approximately $10 \mathrm{~ms}$ after this, a positive wave is seen which is named as P30. Moreover, N9 and N13 waves can also be observed as low-amplitude waves (far-field records), but as having a positive character this time (due to the location relative to the reference electrode).

In another designation, the first wave that is seen or should be seen is called as IP (initial positive) if it is positive, and as IN (initial negative) if it is negative. The subsequent wave is called PI if it is positive, and NI if negative. The following waves are named as NII, PII, NIII, NIV etc.

\subsection{Possible sources of the waves}

\section{Median nerve stimulation}

N9: Brachial plexus activity (action potential).

N11: Spinal medulla dorsal root activity (or dorsal root + cuneate fascicle activity).

N13: Dorsal funiculus activity (post-synaptic grey matter activity).

N14: Lemniscal tract and nucleus activity (lemniscal nucleus and prethalamic lemniscal medial tract activity).

N18: Thalamus posterolateral nucleus activity?

N20: Parietal cortex activity.

P30: Parietal cortex activity.

\section{Tibialis posterior stimulation}

N20: Spinal roots and spinal column activity.

P27: Nucleus gracilis activity.

N35: Parietal cortex activity.

N40: Parietal cortex activity.

Roughly speaking, the response enters spinal column $10 \mathrm{~ms}$ after the stimulus and reaches somesthetic cortex at $20 \mathrm{~ms}$ (it reaches primary cortex in 35-40 ms from the lower extremities).

\subsection{Evaluation}

First criterium to check, is the latency. Latency is the distance between the stimulus and peak of wave in milliseconds.

$$
\text { Latency }=\frac{\text { Distance of the peak of wave from the stimulus }(\mathrm{mm}) \times \text { Sampling duration }(\mathrm{s})}{\text { Sampling length }(\mathrm{mm})}
$$

This is an absolute latency and bilateral comparison is required. However, absolute latency values vary between individuals depending on the length of the extremity or height of a person. Therefore, in addition to absolute latency, "interpeak latency" or "central conduction time" values which are considered even more valuable, are measured.

For example; "N20 - N9", "N20 - N13", "N13 - N9" values obtained by median nerve stimulation are all interpeak latencies. By using this method, absolute latency differences 


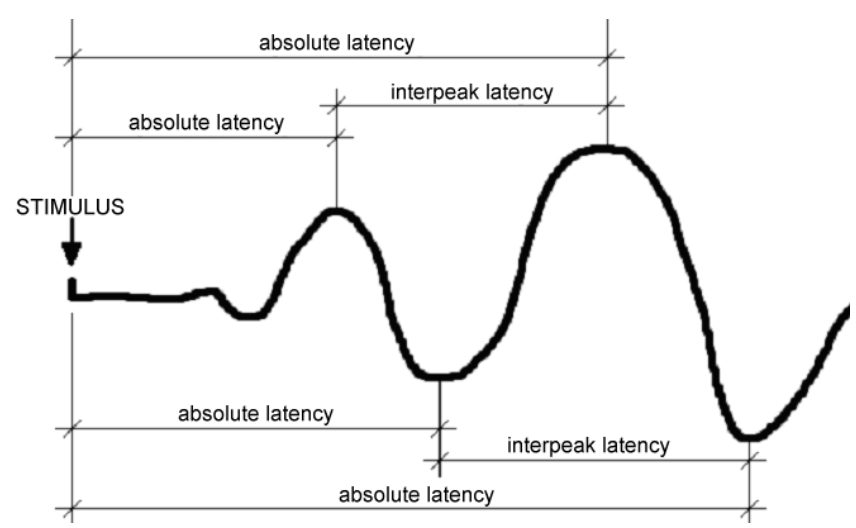

Fig. 11. Absolute \& interpeak latencies.

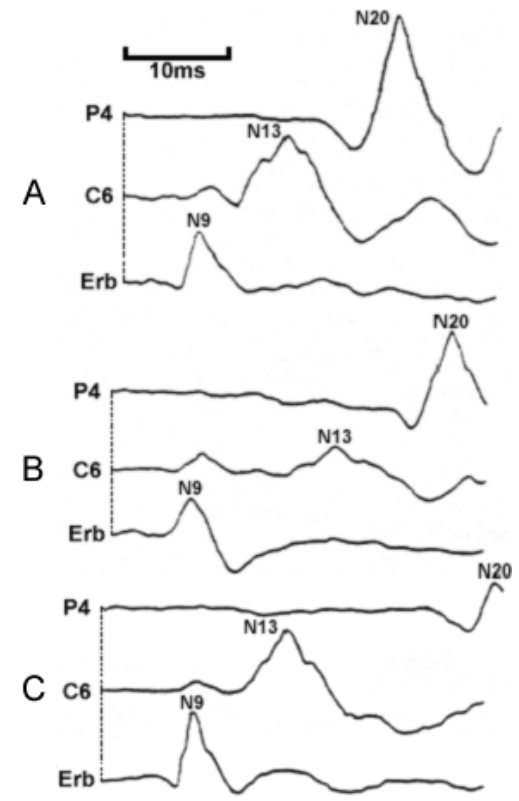

Fig. 12. Evaluation of SSEP recordings. Evaluation of Figure 12A: Amplitudes and waveforms, absolute latencies, and interpeak latencies are normal. Interpretation: Normal individual. Evaluation of Figure 12B: Erb record is normal; N13 absolute latency is prolonged; N13-N9 interpeak latency is prolonged; N13 amplitude is reduced leading to disrupted N11 and N14 notches; N20 absolute latency is prolonged, however, N20-N13 interpeak latency is normal. Interpretation: There is a lesion at cervical spinal cord level, located proximal to the brachial plexus and distal to the brainstem. Evaluation of Figure 12C: Erb and C6 records are normal; C6-Erb relationship is normal; N20 absolute latency is prolonged; N20-N13 interpeak latency is prolonged; N20 amplitude is reduced. Interpretation: There is a disruption in the contralateral tracts between the brainstem and the cortex. 
arising from differences concerning height of individuals or length of extremities, is reduced, and the conduction variability of the peripheral nerve system is partially eliminated.

During evaluation, apart from the "absolute latency" and "central conduction time" values, morphologies and interpeak amplitudes of the waves are examined, as well. However, evaluating amplitudes does not bear much importance because even the same individual may exhibit different amplitude values at different times.

Lastly, the presence or absence of the components and morphologic intercomponent differences, which are also important findings, are evaluated (Figure 12).

In Figure 13, cervical and cortical SSEP recordings of a multiple sclerosis case are shown.

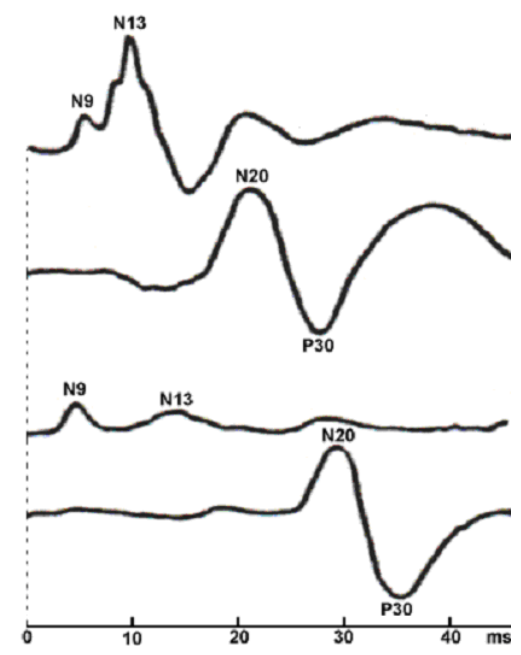

Fig. 13. SSEP changes in multiple sclerosis.

N11, N13, and N14 are weakened in the cervical recording and the interpeak latencies are prolonged. Cortical recording displays preserved N20 and P30 waveforms with prolonged absolute latencies.

Figure 14 is a SSEP recording of a cervical myelopathy case.

All components after N9, appear to be reduced, whereas all the absolute latencies starting from the N9 point are observed to be prolonged.

Evaluation criteria

1. Absolute latencies

2. Interpeak latencies

3. Interpeak amplitude values

4. Presence of components

5. Morphology of the components

6. Intercomponent morphologic differences

7. Bilateral evaluation 


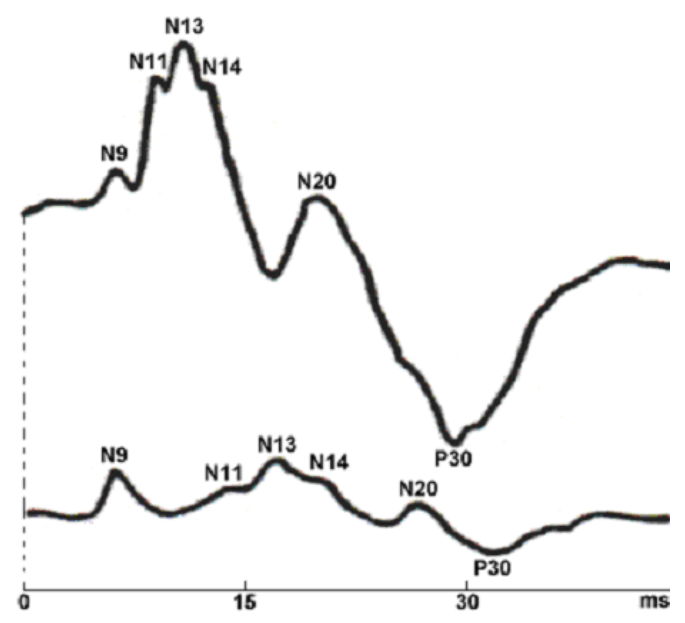

Fig. 14. SSEP changes in cervical myelopathy.

\subsection{Clinical Indications}

SSEP test is applied for checking the peripheral (thick ones) sensory fibers, spinal cord, and somesthetic cortex. It is useful in evaluating and supporting the already established diagnosis concerning the below mentioned cases.

\section{Indications}

- Plexus injuries

- Thoracic outlet syndrome

- Carpal tunnel syndrome

- Tarsal tunnel syndrome

- Evaluation of the peripheral nervous system

- Cervical and back pain

- Musculoskeletal injuries

- Brachial neuritis

- Spinal cord injuries

- Nerve root irritations and traumas

- Neuromuscular diseases

- Neuritis

- Radiculitis

- Motor/sensory deficits

- Vertebral subluxation complex

- Systemic neuropathies

- Lower back pain

- Plexus injuries and irritations

NCV (Nerve Conduction Velocity): It is used for measuring the conduction velocity through the peripheral motor and sensory nerve fibers.

USG (Ultrasonography): Musculoskeletal system ultrasonography of the related areas. 


\subsection{Other indications}

1. Evoked potential tests are indispensable tools for diagnosis and following of multiple sclerosis, a demyelinating disease.

2. It is applied for monitoring purposes in operations involving spinal cord and vertebrae. The recordings in Figure 15 belong to a case of rheumatoid arthritis inducing compression on spinal cord.

3. Can be used in the early diagnosis of sensory problems in newborns.

4. Myelinization can be monitored (Figure 16).
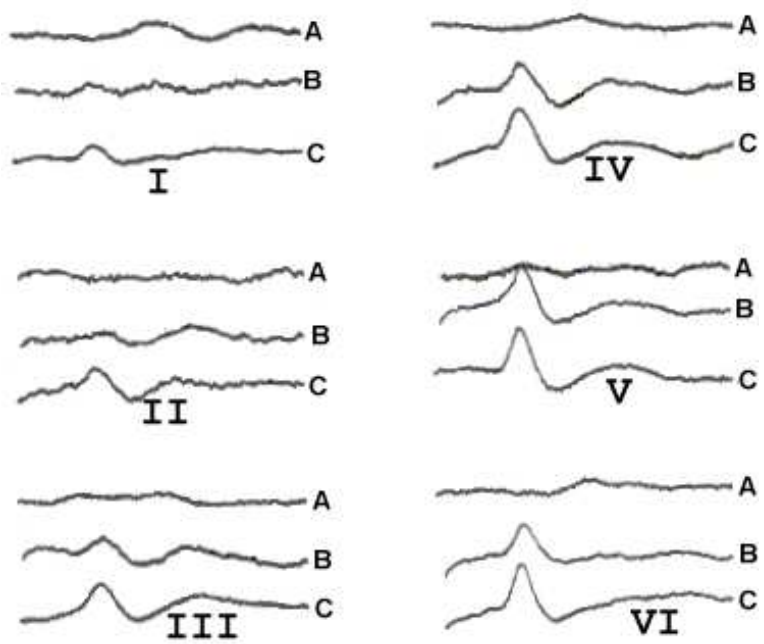

Fig. 15. SSEP changes during spinal cord operation.
A: Tibial nerve stimulation
B: Right ulnar nerve stimulation
I: Beginning of the operation
C: Left ulnar nerve stimulation
II: After left laminectomy
III: After right laminectomy
IV: End of the decompression procedure
$\mathrm{V}$ : Establishing stabilization
VI: End of the operation

The important point here is that patients under general anesthesia may be indifferent to stimuli applied at $3 / \mathrm{s}$ rate. Therefore, during application of SSEP technique for intraoperative monitoring, the stimulus rate should be 1 or 2 stimuli per second. 


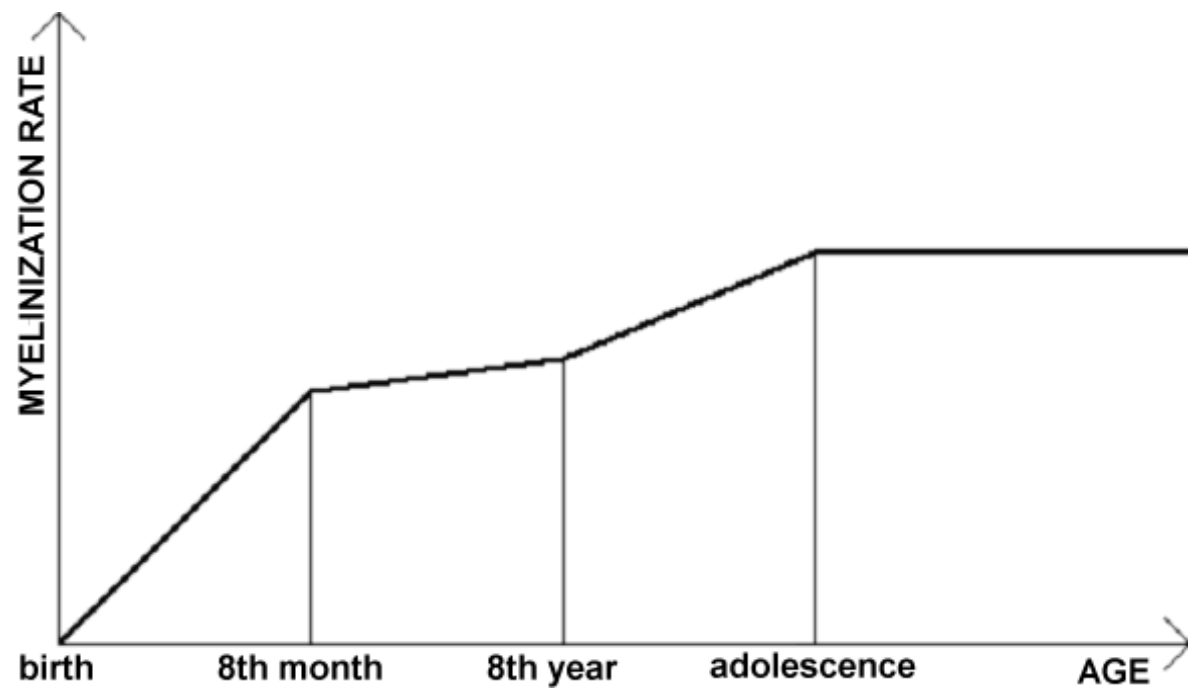

Fig. 16. Myelinization rate.

As shown in Figure 16 obtained from evoked potential recordings, myelinization is very fast during the interval from birth to 8th month, slowing down until the 8th year, and again fast from that point up to the adolescence period.

\section{Auditory evoked potentials}

This is an evoked potential test which is used for checking the auditory canals up to the primary cortex, ie. Heschl gyrus neighboring the temporal lobe, by applying auditory stimulus above hearing threshold to the external auditory canal.

The auditory stimulus is applied through a classic audiometric earphone by delivering a

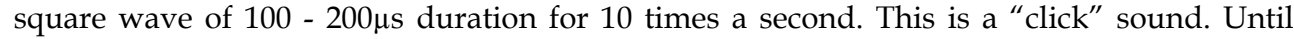
finishing one ear, the other ear is subjected to white noise which is a "hissing" sound containing all frequencies at equal intensity.

The intensity of the sound applied for stimulus is generally above hearing threshold and range betwen 65-70 $\mathrm{dB}$ (in patients with a hearing loss, this value should be increased).

If the stimulus rate is higher than $10 / \mathrm{s}$, absolute latencies and interpeak latencies are prolonged and amplitudes are reduced. When the intensity of the sound is decreased, the wave morphologies change and same events occur (practically, sound intensity is set based on the amplitude of the 5th wave).

For BAEP test, at least 2000 samples of $10 \mathrm{~ms}$ each, should be obtained for each ear, and the test should be repeated at least twice (In clinics, 1024 samples are obtained in normal cases and 4096 samples are obtained in pathologic cases. Sampling of both are repeated twice).

As mentioned before, a band-pass filter within range of 100-3000 Hz should be used. Again, as previously noted, the required amplification is a large one $(500,000 \mathrm{X})$, because the average amplitude of BAEP components are at $0.25 \mu \mathrm{V}$ level. 
Active electrode is the vertex electrode $(\mathrm{Cz})$. Reference electrode is the one at the ipsilateral ear lobe or ipsilateral mastoid bone. It may be better if the mastoid electrodes are not used because they may lead to muscle artifacts. In this case, the electrodes used in BAEP recording will be as follows: $\mathrm{Cz}-\mathrm{A} 1-\mathrm{A} 2$

The first thing to be done during recording is to monitor the first BAEP component (wave 1). If this wave can not be discerned, then the following steps can be taken:

1. Intensity of the sound is increased.

2. Poles of the earphone speaker are switched.

3. Stimulus rate $(10 / \mathrm{s})$ is reduced.

4. Reference electrode at the ear lobe is placed at the external ear canal.

5. In presence of unavoidable muscular noise, the patient is subjected to sedation.

Moreover, it should be born in mind that:

a. People above 60 years of age exhibit significantly prolonged latency.

b. Interpeak latencies are shorter in women than in men.

c. Last two of the BAEP components (there are 7 in total), which are named as "wave VI" and "wave VII", may never be seen.

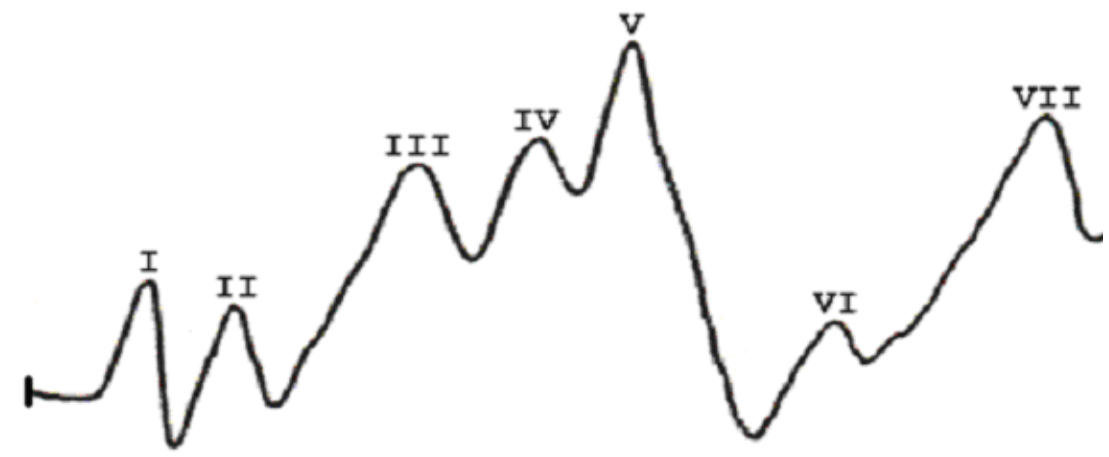

Fig. 17. Waveforms seen in BAEP recording.

Although the activated anatomic locations are completely certain, sources of the waves obtained by BAEP are not obvious. They may be as follows:

i. Distal action potential of the acoustic nerve (negative wave in the ipsilateral ear electrode).

Peripheral

1.3 ms latency

ii. Ipsilateral proximal acoustic nerve and/or cochlear nucleus activity.

Bulbar

2.4 ms latency

iii. Ipsilateral superior nucleus olivarius activity.

Pontine

3.3 ms latency

iv. Lateral lemniscal nuclear or axonal activity.

Pontine 
4.4 ms latency

v. Inferior colliculus activity.

Mesencephalic

5.2 ms latency

vi. Medial geniculate body activity.

Thalamic

6.5 ms latency

vii. Thalamo-cortical projection activity.

Cortical

8.5 ms latency

In Figure 18, we see a normal BAEP recording with 5 components which has no pathology despite absence of two components.

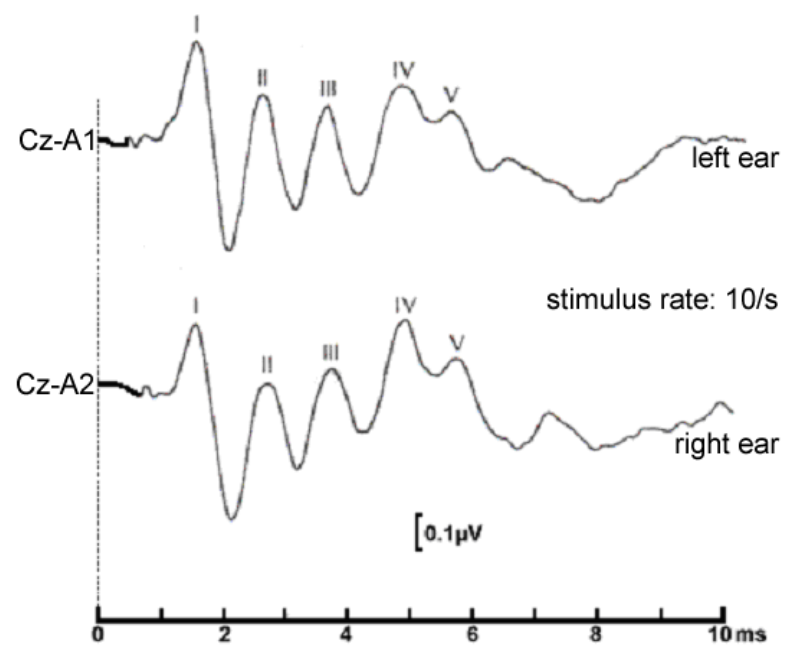

Fig. 18. Normal BAEP recording.

\subsection{Indications}

1. 1. Hearing loss, hearing imbalance

2. Balance disorders

3. Tinnitus

4. Assessment of type and level of hearing loss in children below 5 years of age

5. Metabolic, demyelinating, degenerative diseases and tumors of the brainstem

6. Ear canal lesions outside the brainstem

7. Monitorization during operations concerning brainstem

8. Control and follow-up after operations concerning brainstem

9. Headaches

10. Head traumas

11. Hyperflexion/hyperextension

12. Comas (with EEG). In Figure 19, you can see various coma levels reflected in BAEP recording. 


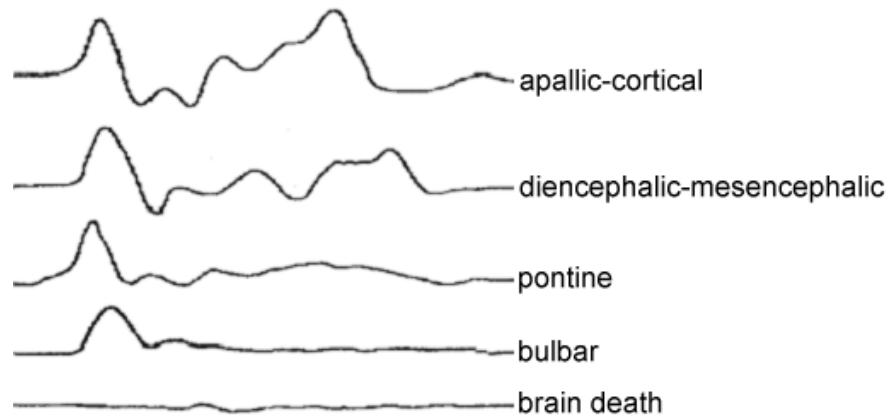

Fig. 19. Coma levels in BAEP recordings. It is noteworthy that as the coma deepens, BAEP components disappear from proximal to distal.

During the BAEP test, component amplitudes are not taken into consideration because amplitude values are of considerably variable nature. The most common pathology seen during the test is prolonged "I-III" or "I-V" intervals. In Figure 20, you can see a BAEP recording of an acoustic neurinoma case, causing a prolonged " $\mathrm{I}-\mathrm{V}$ " interval.

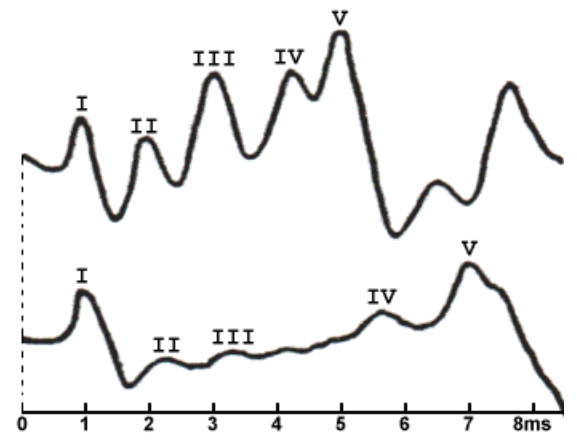

Fig. 20. BAEP changes in acoustic neurinoma.

We have to underscore once again that BAEP tests are not influenced by anesthetics, barbiturates, and attention-consciousness-sleep statuses.

\section{Visual evoked potentials}

Visual evoked potentials are obtained from the optic tract by recording the evoked potentials generated by retinal stimulation. The sources of the stimulus are as follows:

1. Stroboscopic flash light with regular flashing intervals: Applied on babies and uncooperative patients.

2. Flashing LED: Applied as an intraoperative stimulus source. However, it requires use of specially designed lenses.

3. Alternating checkerboard pattern stimulation: This is the stimulation method used most commonly and it is a more sensitive and stable technique. VEP test performed by applying this stimulation is also called as PSVEP (Pattern-Shift Visual Evoked Potential). 
Testing environment should be dark, or at least dim. The patient sits in front of a 70-100 cm distant $\mathrm{B} / \mathrm{W}$ monitor with a checkerboard pattern showing constant luminance and he/she continuously and carefully looks at this pattern. The checks alternate from black to white and from white to black at every 1 or 2 seconds. Each alternation acts as a stimulation and generates an evoked potential at the occipital lobe.

The size of the squares on the checkerboard pattern should be between $28^{\prime}-31^{\prime}$ (practically, squares with a size of $30^{\prime}$, ie. $0.5^{\circ}$, are commonly used). In patients with a reduced visual acuity, this size should be increased a little. It should be borne in mind that as the size changes, the latency and amplitude of the P100 wave changes, as well. As shown in Figure 21 , as the squares get smaller, the latency is prolonged and amplitude is elevated.

Note: The polarities of the VEP curves shown in Figure 21 were placed in reverse order, meaning that P100 wave, which should have a lower position, was positioned upwards due to location of the positive potential.

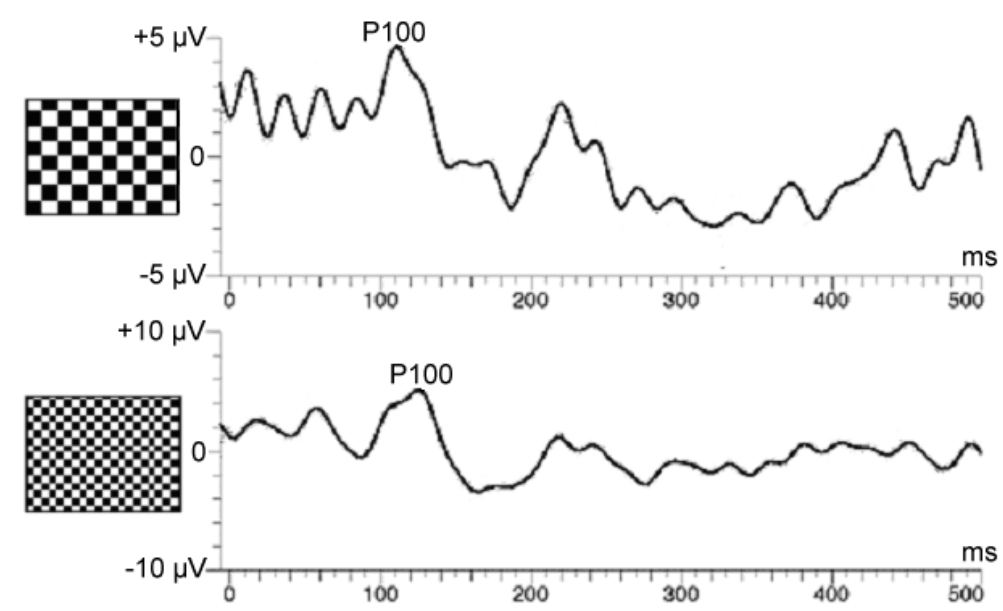

Fig. 21. The size of squares effects P100 quality.

One should also know that if the stimulus rate (normally 1 or 2 stimuli per second) is increased, then subsequently latency is prolonged. Moreover, latency and amplitude values are affected by factors such as brightness of the room, luminance of the screen, and visual fixation of the patient.

As mentioned before, average amplitude of VEPs is about $5-10 \mu \mathrm{V}$ and it is enough for $10.000 \mathrm{x}$ amplification. In other words, this is the type of evoked potential that can be recorded most easily.

In VEP recording, active electrode is $\mathrm{Oz}$, whereas reference electrode is oftenly $\mathrm{Fz}$, and sometimes A1-A2. Routine test is always performed unilaterally (monocular). However, since this evaluation does not work in pathologies past the optic chiasm, binocular VEP test is carried out and the number of active electrodes over the occipital region is 5 in this test. 


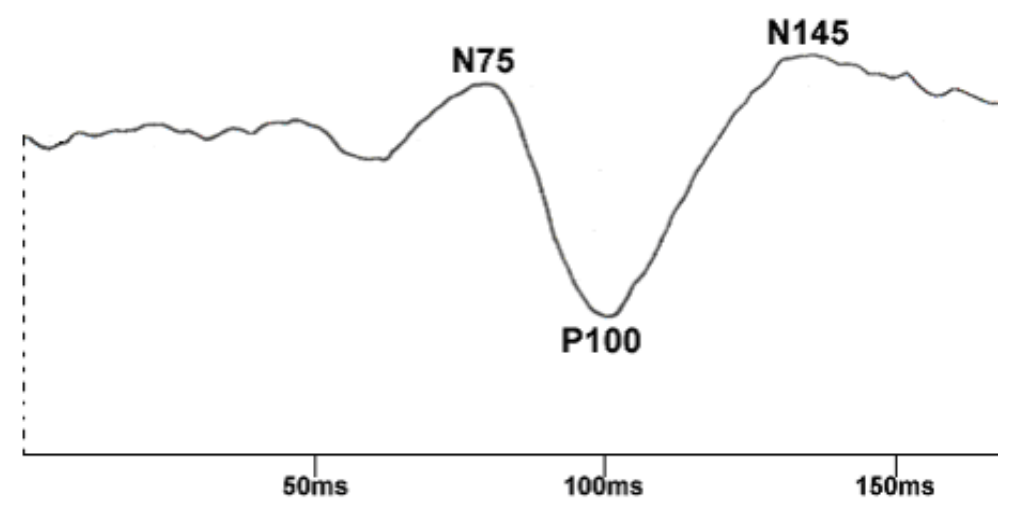

Fig. 22. Components of the VEP.

\subsection{Evaluation}

As seen in Figure 22, since N75 and N145 show high variability during 3-component VEP recording, only P100 wave, which is the essential component, is evaluated (VEP test is also called as "P100 test").

Bilateral comparison bears great importance in the evaluation. However, first, latency and amplitude values of the P100 wave should be identified.

It should be taken into consideration that women have a $5 \mathrm{~ms}$ shorter average P100 latency compared with the men, and that among people above 60 years of age, latency may reach high values such as $125 \mathrm{~ms}$.

When there is an amplitude difference exceeding $2 \mu \mathrm{V}$ between the VEP recordings of two eyes which can not be explained in terms of technical factors, it is a remarkable finding. However, one should always remember that abnormal amplitudes are almost always observed with abnormal latencies.

Main pathologies that may be seen in VEP are as follows.

1. Latency may be prolonged, but amplitude and form may remain normal. Multiple sclerosis, a demyelinating disease, is reflected on VEP in this fashion.

Figure 23 is the VEP recording of a patient with a prolonged P100 absolute latency in the left eye due to multiple sclerosis. 


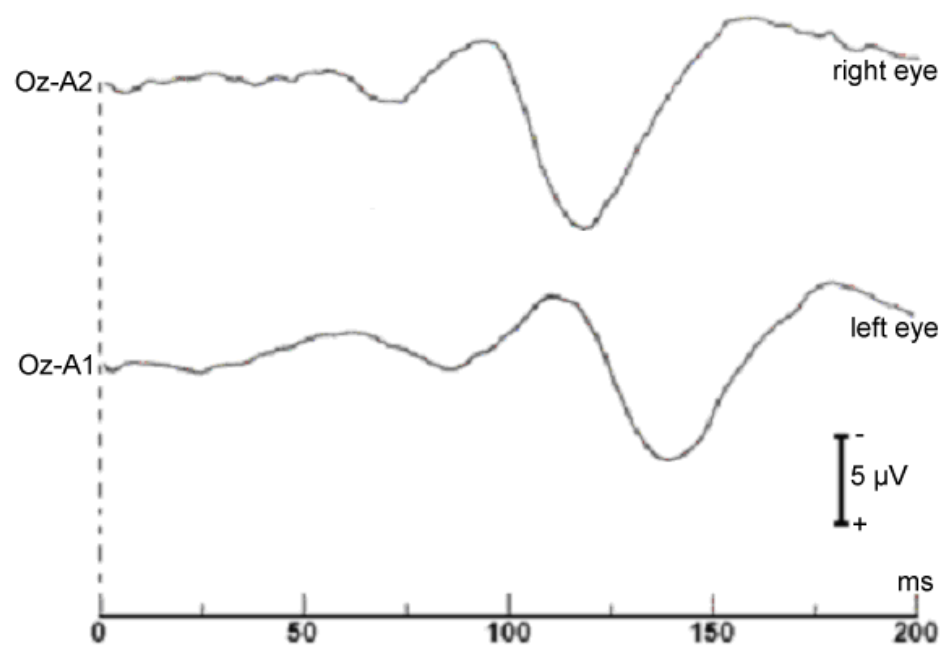

Fig. 23. Prolonged latency: Multiple sclerosis in left eye.

Even in the absence of visual complaints, $30 \%$ of multiple sclerosis patients display VEP pathology.

2. Amplitude may be reduced. Lesions compressing the optical nerve are reflected in the VEP recording in this fashion. However, amplitude may be reduced in refractive abnormalities, cataract, and retinopathy, as well. Moreover, even a decrease in the attention of the patient can lead to amplitude reduction.

You can see the prolonged latency and amplitude reduction in the VEP recording of a patient with right optic neuritis (Figure 24).

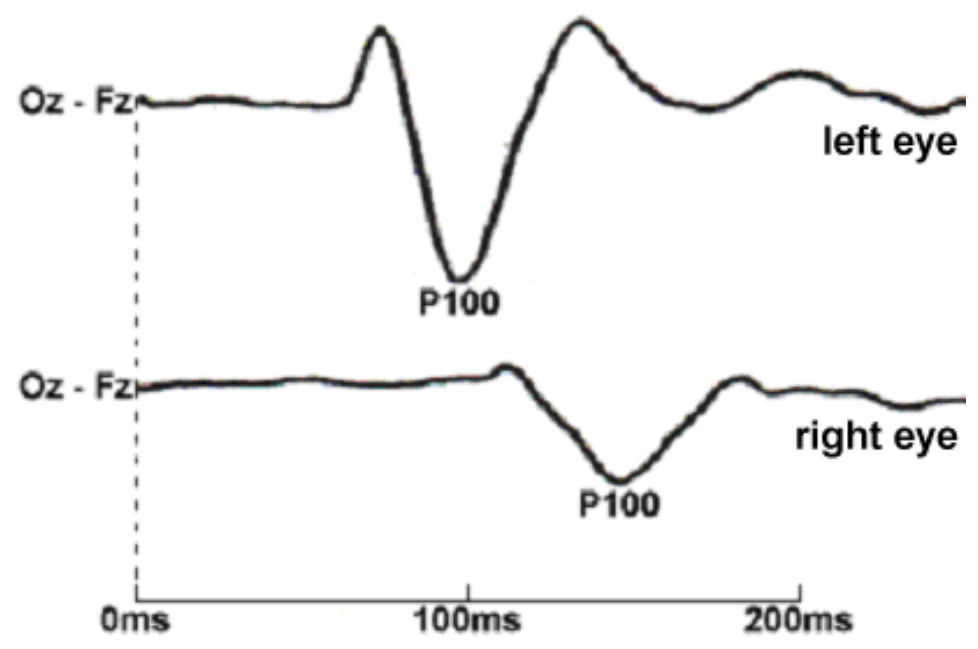

Fig. 24. Reduced amplitude \& prolonged latency: Optic neuritis in right eye. 
3. In cases involving compressive lesions, amplitude reduction may cause changes in the form, as well. Even in people with no complaints, tumors located at the optic tracts may lead to loss of N75 and prolonged P100.

\subsection{Indications}

1. Optic nerve damage

2. Optic neuritis

3. Headache

4. Head trauma

5. Brain aneurysm

6. Brain tumors

7. Blurred vision

8. Intraoperative monitoring

It may even be used in operations which do not involve optic tracts, but might influence them. However, as shown in Figure 25, the effect of general anesthesia over VEP is considerably high.

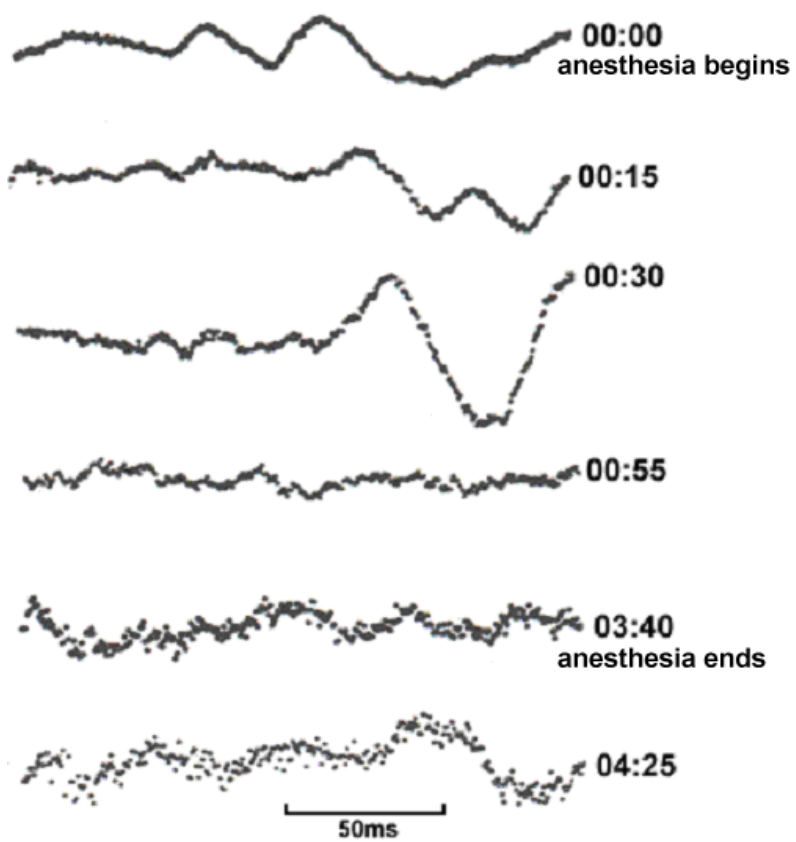

Fig. 25. Effect of general anesthesia on the VEP. 


\section{Conclusion}

Which conditions indicate evoked potential tests?

1. Persistence of symptoms despite decided and ongoing treatment

2. Presence of subjective complaints without supportive objective findings

3. Negative X-ray, CT, MRI, or EMG results despite existing complaints

4. Presence of radicular complaints

5. Existing nerve irritation or damage requiring definition

6. Need for advanced diagnosis/treatment

7. Cases requiring confirmation of presence of pain

Advantages and disadvantages of the evoked potential tests

\section{Pros}

1. Although modern imaging methods other than PET can depict pathological localizations in detail, they can not provide data on functional/physiological structures (ie. metabolic cerebral diseases). Thus, evoked potential tests compensate for this shortcoming.

2. Non-invasive character

3. Objective measurement

4. Subcortical components of auditory and somatosensory evoked potentials are not influenced by general anesthesia, sleep, and states of consciousness.

5. Significantly low cost compared with the modern imaging modalities.

\section{Cons}

1. There is still no standard in technical regard. Even the fundamental terminological standards have not been completed yet, let alone being a "gold standard". However, currently; SSEP, BAEP, and VEP techniques have been almost standardized.

2. Tests are long and tedious for patients

3. The risk of technical error, therefore the likelihood of repeating the test, is high

4. While the characteristics of the acquired recordings are mostly known, their sources and mechanisms have not yet been completely or clearly understood (due to the complexity of the brain anatomy and nonlinear nature of the brain physiology).

\section{References}

Anbar, Michael. \& Spangler Robert. A. \& Scott Peter. (1985). Clinical Biophysics, Warren H. Green, ISBN 0-87527-316-5, Missouri, USA

Aston, Richard. (1991). Principles of Biomedical Instrumentation and Measurement, Macmillan Publishing Company, ISBN 0-02-946562-1, New York, USA

Carr, Joseph J. \& Brown, John M. (2001). Introduction to Biomedical Equipment Technology (4th edition), Prentice Hall, ISBN 0-13-010492-2, New Jersey, USA

Chiappa, Keith H. (1997). Evoked Potentials in Clinical Medicine (3rd edition), LippincottRaven Publishers, ISBN 0-397-51659-2, Philadelphia, USA 
Daube, Jasper R. (1996). Clinical Neurophysiology, F. A. Davis company, ISBN 0-8036-0073-9, Philadelphia, USA

Kimura, Jun. (2001). Electrodiagnosis in Diseases of Nerve and Muscle: Principles and Practice (3rd edition), Oxford University Press, ISBN 0-19-512977-6, New York, USA

Misulis, Karl E. \& Fakhoury, Toufic. (2001). Spehlmann's Evoked Potential Primer (3rd edition), Butterworth-Heinemann, ISBN 0-7506-7333-8, Maryland, USA

Webster, John G. (2004). Bioinstrumentation, John Wiley \& Sons, ISBN 0-471-45257-2, Maryland, USA 


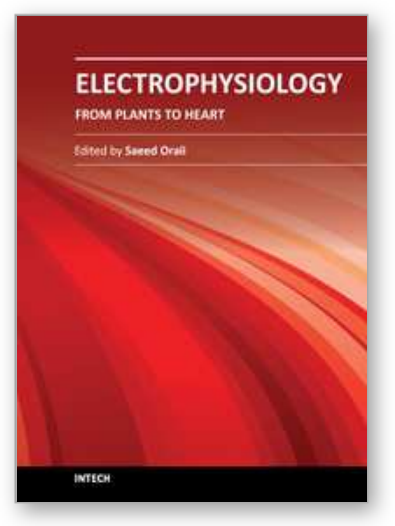

\author{
Electrophysiology - From Plants to Heart \\ Edited by Dr. Saeed Oraii
}

ISBN 978-953-51-0006-5

Hard cover, 202 pages

Publisher InTech

Published online 03, February, 2012

Published in print edition February, 2012

The outstanding evolution of recording techniques paved the way for better understanding of electrophysiological phenomena within the human organs, including the cardiovascular, ophthalmologic and neural systems. In the field of cardiac electrophysiology, the development of more and more sophisticated recording and mapping techniques made it possible to elucidate the mechanism of various cardiac arrhythmias. This has even led to the evolution of techniques to ablate and cure most complex cardiac arrhythmias. Nevertheless, there is still a long way ahead and this book can be considered a valuable addition to the current knowledge in subjects related to bioelectricity from plants to the human heart.

\title{
How to reference
}

In order to correctly reference this scholarly work, feel free to copy and paste the following:

Ahmet Akay (2012). Evoked Potentials, Electrophysiology - From Plants to Heart, Dr. Saeed Oraii (Ed.), ISBN: 978-953-51-0006-5, InTech, Available from: http://www.intechopen.com/books/electrophysiology-from-plantsto-heart/evoked-potentials

\section{INTECH}

open science | open minds

\section{InTech Europe}

University Campus STeP Ri

Slavka Krautzeka 83/A

51000 Rijeka, Croatia

Phone: +385 (51) 770447

Fax: +385 (51) 686166

www.intechopen.com

\section{InTech China}

Unit 405, Office Block, Hotel Equatorial Shanghai

No.65, Yan An Road (West), Shanghai, 200040, China

中国上海市延安西路65号上海国际贵都大饭店办公楼 405 单元

Phone: +86-21-62489820

Fax: +86-21-62489821 
(C) 2012 The Author(s). Licensee IntechOpen. This is an open access article distributed under the terms of the Creative Commons Attribution 3.0 License, which permits unrestricted use, distribution, and reproduction in any medium, provided the original work is properly cited. 